\title{
Cauchy Problems for Discrete Holomorphic Functions
}

\author{
Christer Oscar Kiselman ${ }^{1}$ iD
}

Received: 17 April 2020 / Accepted: 12 August 2020 / Published online: 2 November 2020

(c) The Author(s) 2020

\begin{abstract}
We solve Cauchy problems for discrete holomorphic functions defined on the Gaussian integers, which leads to the existence of discrete holomorphic functions with arbitrarily fast growth. This proves that certain classes of functions are closed in the sense of mathematical morphology.
\end{abstract}

Keywords Discrete holomorphic functions - Duality of convolution operators · Mathematical morphology

\section{Introduction}

\subsection{Discrete Holomorphic Functions}

Functions on the ring of Gaussian integers $\mathbf{Z}[\mathrm{i}]=\mathbf{Z}+\mathrm{i} \mathbf{Z} \subset \mathbf{C}$ that satisfy a discrete convolution equation corresponding to the Cauchy-Riemann equation have been studied since the 1940s. Rufus Philip Isaacs [6,7] developed a theory for these functions. He called them monodiffric, meaning that the quotients of differences in two different directions are equal. Those for the direction from 0 to 1 versus the direction from 0 to i (illustrated by the arrows $\uparrow$ ) he called monodiffric of the first kind; those for the direction from 0 to $1+\mathrm{i}$ versus the orthogonal direction from 1 to $\mathrm{i}$ (illustrated by the arrows $($ ) he called monodiffric of the second kind. He expressed the opinion that the latter "seemed less promising than the present course" [7: 258]. I therefore think that

Dedicated to the memory of Carlos Alberto Berenstein.

Communicated by Daniel Aron Alpay.

This article is part of the topical collection "In memory of Carlos A. Berenstein (1944-2019)" edited by Irene Sabadini and Daniele Struppa.

Christer Oscar Kiselman

kiselman@it.uu.se; christer@kiselman.eu

http://www.cb.uu.se/ kiselman

1 Department of Information Technology, Uppsala University, P. O. Box 337, 75105 Uppsala, Sweden

Birkhäuser 
it is justifiable to associate the monodiffric functions of the first kind with the name of Isaacs, as I did in my paper [9]. More recent research on this class includes that of Nakamura and Rosenfeld [17].

The monodiffric functions of the second kind were studied by Jacqueline Ferrand (1944) [5], which is the reason why I associated them with her name in my paper (2008) [10]. She called these functions préholomorphes. Later studies on this class include those by Duffin (1956) [4], Lovász (2000) [15], Kenyon (2000) [8] and Benjamini and Lovász (2000) [1].

For these two most studied classes, we shall prove that a Cauchy problem has a unique solution: for those of the first kind with data given on

$$
\{z \in \mathbf{Z}[\mathrm{i}] ; \operatorname{Im} z=0\} \cup\{z \in \mathbf{Z}[\mathrm{i}] ; \operatorname{Re} z=0 \text { and } \operatorname{Im} z \leqslant 0\}
$$

for those of the second kind with data given on the axes:

$$
\mathbf{Z} \cup \mathrm{i} \mathbf{Z}=\{z \in \mathbf{Z}[\mathrm{i}] ; \operatorname{Im} z=0\} \cup\{z \in \mathbf{Z}[\mathrm{i}] ; \operatorname{Re} z=0\}
$$

For the question discussed here the functions of the second kind are more symmetric than those of the first kind and require less work-you can compare Propositions 7.4 and 8.4 .

\subsection{Duality of Convolution Operators}

Duality is a term which represents a collection of ideas where two sets of mathematical objects confront each other. A most successful duality is that between the space $\mathscr{D}(\Omega)$ of test functions (smooth functions of compact support) and its dual $\mathscr{D}^{\prime}(\Omega)$ of distributions.

Similarly, the theory of analytic functionals, developed by André Martineau in his doctoral thesis [16]—also in (Euvre de André Martineau [18]: 47-210)—is based on a duality, in many but not all respects analogous to distribution theory.

In complex geometry, lineal concavity and lineal convexity can be treated successfully using concepts of duality (see my publication 2019 [11] and my manuscript [12]).

In this paper we shall not treat these instances of duality but instead take a look at discrete versions of the Cauchy-Riemann operator.

Questions on duality gave rise to the study of Cauchy problems mentioned in Sect. 1.1.

\subsection{Mathematical Morphology}

A relatively new branch of science, mathematical morphology, can be quite helpful in providing guiding concepts and ideas in the study of discrete convolution operators and related topics, like discrete optimization. In fact, these ideas led to the questions on duality alluded to in Sect. 1.2. For more on mathematical morphology, see Serra [19], Serra, Ed. [20] and my manuscript (MS [13]). 
We shall apply ideas from mathematical morphology to the duality of convolution operators, in particular those that define monodiffric functions.

\section{Notation}

We use the common symbols $\mathbf{N}=\{0,1,2, \ldots\}, \mathbf{Z}, \mathbf{R}, \mathbf{C}$ for the semiring of natural numbers, the ring of integers, the fields of real and complex numbers, respectively. The Gaussian integers $\mathbf{Z}[\mathrm{i}]=\mathbf{Z}+\mathrm{i} \mathbf{Z}$ constitute a subring of $\mathbf{C}$.

The family of all subsets of a set $X$ is called the power set of $X$ and will denoted by $\mathscr{P}(X)$. Thus $A \in \mathscr{P}(X)$ if and only if $A \subset X$. This set is ordered by the relation that $A \leqslant B$ if and only if $A \subset B$. We denote by $\mathscr{P}_{\text {finite }}(X)$ the family of all finite subsets of $X$.

Following Bourbaki ([2]: Chapter II, §5, No.1, p. 101) we shall denote the set of all mappings from $X$ into $Y$ by $\mathscr{F}(X, Y)$. If $H$ is an abelian semigroup with zero 0 , we define the support of a function $f: X \rightarrow H$ as the set where $f$ is nonzero. We denote by $\mathscr{F}_{\text {finite }}(X, H)$ the subset of $\mathscr{F}(X, H)$ consisting of all functions which are zero outside a finite subset of $X$.

We write $l^{p}(X), 1 \leqslant p \leqslant \infty$, for the space of all functions $f: X \rightarrow \mathbf{C}$ such that $\sum_{x \in X}|f(x)|^{p}<+\infty(1 \leqslant p<\infty)$ and $\sup _{x \in X}|f(x)|<+\infty(p=\infty)$. They are equal if and only if $X$ is finite; then $l^{p}(X)=\mathscr{F}(X, H)=\mathscr{F}_{\text {finite }}(X, H)$ for all $p$.

\section{Convolution}

Definition 2.1 If $G$ is an abelian semigroup we define the convolution product $h=$ $f * g$ of two functions $f, g$ defined on $G$ and with real or complex values by the formula

$$
h(z)=\sum_{\substack{x, y \in G \\ x+y=z}} f(x) g(y), \quad z \in G,
$$

provided the sum can be given a meaning.

The semigroup $G$ here will most often be the group $\mathbf{Z}$ [i], but we shall also consider other groups.

If one of $f, g$ is nonzero only in a finite subset of $G$, the sum is always well defined, but we need to consider more general situations.

The Kronecker delta $\delta_{a}$ is the function which takes the value 1 at $a$ and is zero elsewhere. For $a=0$ we get a neutral element: $f * \delta_{0}=f$ for all functions $f$.

As shown for instance in my texts (MS [12]: Example 5.2) and (MS [13]: Example 13.2.1), we cannot hope for a general convolution algebra, but we can confront a family of functions with another family, which is an instance of duality. The simplest example of this is that $\mathscr{F}_{\text {finite }}(G, \mathbf{C})$ can work against $\mathscr{F}(G, \mathbf{C})$ and conversely. But there are several other cases of interest.

Provided that we accept $+\infty$ as a value, we can define the convolution product $|f| *|g|$ of the absolute values of any two functions $f, g \in \mathscr{F}(G, \mathbf{C})$. The sum is 
simply defined to be the supremum of all finite partial sums:

$$
(|f| *|g|)(z)=\sup _{A \in \mathscr{P}_{\text {finite }}(G)} \sum_{\substack{x, y \in A \\ x+y=z}}|f(x)| \cdot|g(y)|
$$

and takes its value in $[0,+\infty]$. Therefore it makes sense to require $|f| *|g|$ to be finite everywhere. If this is so, then also $f * g$ is well defined as a function with finite values; the sum defining it being absolutely convergent at every point. More precisely, we have the following result concerning commutativity and associativity.

Proposition 2.2 Let $G$ be an abelian group and $f_{j}: G \rightarrow \mathbf{C}, j=1,2,3$, three functions.

1. If $\left|f_{1}\right| *\left|f_{2}\right|$ is finite everywhere, then $f_{1} * f_{2}$ is defined everywhere as an absolutely converging series, $f_{1} * f_{2}=f_{2} * f_{1}$, and $\left|f_{1} * f_{2}\right| \leqslant\left|f_{1}\right| *\left|f_{2}\right|$.

2. If $\left(\left|f_{1}\right| *\left|f_{2}\right|\right) *\left|f_{3}\right|$ is finite everywhere, then $\left(f_{1} * f_{2}\right) * f_{3}$ and $f_{1} *\left(f_{2} * f_{3}\right)$ are defined everywhere as absolutely converging series, and they are equal.

The proof of this proposition appears in my manuscripts (MS [12]: Proposition 5.3) and (MS [13]: Proposition 13.2.2) and is not difficult at all.

\section{Duality in Convolution}

Definition 3.1 Given an abelian semigroup $G$ and any subfamily $\mathscr{G}$ of $\mathscr{F}(G, \mathbf{C})$, we define

$$
\Gamma(\mathscr{G})=\{f: G \rightarrow \mathbf{C} ; \text { for all } g \in \mathscr{G},|f| *|g|<+\infty\},
$$

thus defining a transformation $\Gamma: \mathscr{P}(\mathscr{F}(G, \mathbf{C})) \rightarrow \mathscr{P}(\mathscr{F}(G, \mathbf{C}))$.

If $G$ is finite, we have $\Gamma(\mathscr{G})=\mathscr{F}(G, \mathbf{C})$.

Definition 3.2 We define a mapping $\Psi$ by

$$
\Psi(\mathscr{G})=\{f \in \Gamma(\mathscr{G}) ; \text { for all } g \in \mathscr{G}, f * g=0\}, \quad \mathscr{G} \subset \mathscr{F}(G, \mathbf{C}),
$$

where the formula defines $\Psi: \mathscr{P}(\mathscr{F}(G, \mathbf{C})) \rightarrow \mathscr{P}(\mathscr{F}(G, \mathbf{C}))$.

Clearly $\Gamma$ and $\Psi$ are decreasing, so that the compositions $\Gamma \circ \Gamma, \Psi \circ \Gamma, \Gamma \circ \Psi, \Psi \circ \Psi$ are all increasing. Together with the fact that $\Psi \leqslant \Gamma$ these facts give rise to order relations

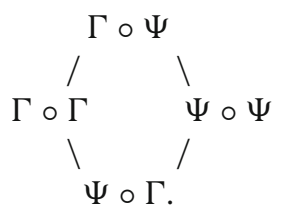


The smallest of these compositions is $\Psi \circ \Gamma$ as shown, and it maps all families to the singleton set of the zero function and is therefore idempotent. The composition $\Gamma \circ \Psi$ is in general not idempotent.

It can happen that $\Psi^{\circ 2}(\mathscr{G})$ contains $\Gamma^{\circ 2}(\mathscr{G})$ strictly (Examples 3.9 and 3.11) as well as it being strictly contained in $\Gamma^{\circ 2}(\mathscr{G})$ (Example 3.10).

A mapping $\eta: X \rightarrow X$, where $X$ is a preordered set, is called an ethmomorphism if it is increasing and idempotent. An ethmomorphism $\kappa$ is said to be a cleistomorphism if it is larger than the identity. A fixed point $x$ for $\kappa$, thus an element $x \in X$ such that $\kappa(x)=x$, is said to be closed for $\kappa$.

Given two preordered sets $X$ and $Y$, a Galois correspondence is a pair $(F, G)$ of mappings, $F: X \rightarrow Y$ and $G: X \rightarrow Y$, which are both decreasing and such that the two compositions $G \circ F: X \rightarrow X$ and $F \circ G: Y \rightarrow Y$ are larger than the identity mappings. Both $G \circ F$ and $F \circ G$ are increasing. It follows that they satisfy $F \circ G \circ F=F$ and $G \circ F \circ G=G$ and so are cleistomorphisms. See (Kuroš [14]) and (Cohn [3]) for general results on Galois correspondences.

The pairs $(\Gamma, \Gamma)$ and $(\Psi, \Psi)$ are Galois correspondences as we shall now prove.

Proposition 3.3 The mapping $\Gamma$ is decreasing and satisfies

$$
\bigcap_{j \in J} \Gamma\left(\mathscr{G}_{j}\right)=\Gamma\left(\bigcup_{j \in J} \mathscr{G}_{j}\right)
$$

for all families $\left(\mathscr{G}_{j}\right)_{j \in J}$, from which is follows that id $\leqslant \Gamma \circ \Gamma$. As a consequence, the pair $(\Gamma, \Gamma)$ is a Galois correspondence.

Similarly for $\Psi$.

Proof As already noted, $\Gamma$ is decreasing, and like all decreasing mappings it satisfies $\bigcap_{j} \Gamma\left(\mathscr{G}_{j}\right) \supset \Gamma\left(\bigcup_{j} \mathscr{G}_{j}\right)$. The inclusion in the other direction follows from the definition-in fact both inclusions are easily seen to hold from the definition.

If $|g| *|h|<+\infty$, then $g \in \Gamma(\{h\})$. If $|g| *|h|<+\infty$ for all $h \in \mathscr{H}$, then $g \in \bigcap_{h \in \mathscr{H}} \Gamma(\{h\})=\Gamma\left(\bigcup_{h \in \mathscr{H}}\{h\}\right)=\Gamma(\mathscr{H})$. If $|g| *|h|<+\infty$ for all $g \in \mathscr{G}$ and all $h \in \mathscr{H}$, then $\mathscr{G} \subset \Gamma(\mathscr{H})$. Finally, taking $\mathscr{H}=\Gamma(\mathscr{G})$, we get $\mathscr{G} \subset \Gamma(\Gamma(\mathscr{G}))$, proving id $\leqslant \Gamma \circ \Gamma$.

We shall investigate whether some classes of functions are closed for the corresponding cleistomorphisms, in particular for $\Gamma \circ \Gamma$ and $\Psi \circ \Psi$.

\subsection{Adding Functions of Finite Support}

Proposition 3.4 The image $\Gamma(\mathscr{G})$ of an arbitrary family $\mathscr{G}$ of functions is a vector subspace of $\mathscr{F}(G, \mathbf{C})$ which contains $\mathscr{F}_{\text {finite }}(G, \mathbf{C})$. We shall use the notation

$$
\mathscr{G}^{[+]}=\mathscr{G}+\mathscr{F}_{\text {finite }}(G, \mathbf{C})=\left\{g+\rho ; g \in \mathscr{G} \text { and } \rho \in \mathscr{F}_{\text {finite }}(G, \mathbf{C})\right\} .
$$

Thus $\Gamma\left(\mathscr{G}^{[+]}\right)=\Gamma(\mathscr{G})$, which implies that $\mathscr{G} \subset \mathscr{G}^{[+]} \subset \Gamma^{\circ 2}(\mathscr{G})$ and that

$$
\Gamma(\mathscr{G})=\Gamma\left(\mathscr{G}^{[+]}\right)=\Gamma\left(\Gamma^{\circ 2}(\mathscr{G})\right)=\Gamma^{\circ 3}(\mathscr{G}) .
$$


Proof This is easy so see.

We note the following easy consequence.

Corollary 3.5 The operation $\Gamma$ can be defined on the quotient space $\mathscr{F}(G, \mathbf{C}) / \mathscr{F}$ finite $(G, \mathbf{C})$.

This quotient space is analogous to the space of singularities $\mathscr{D}^{\prime}(\Omega) / \mathscr{E}(\Omega)$, the space of distributions modulo the subspace of smooth functions. Also the more elementary singularity space $C^{0}(\Omega) / C^{\infty}(\Omega)$ is interesting-these spaces deserve being studied, I believe.

\subsection{Forming Convolutions with Functions of Finite Support}

Proposition 3.6 If $\mathscr{G}$ is a subset of $\mathscr{F}_{\text {finite }}(\mathbf{C}, \mathbf{C})$, the second dual $\Gamma(\Gamma(\mathscr{G}))=\Gamma^{\circ 2}(\mathscr{G})$ always contains the set of all convolution products $g * \rho, g \in \mathscr{G}$, where $\rho$ is an arbitrary element of $\mathscr{F}_{\text {finite }}(G, \mathbf{C})$ :

$$
\Gamma^{\circ 2}(\mathscr{G}) \supset\left\{g * \rho ; g \in \mathscr{G} \text { and } \rho \in \mathscr{F}_{\text {finite }}(G, \mathbf{C})\right\}=\mathscr{G}^{[*]},
$$

where the last equality defines the family $\mathscr{G}[*] \supset \mathscr{G}$.

Proof If $h=g * \rho$ with $g \in \mathscr{G}$ and $\rho$ has finite support, then as in all convolution products with three factors where only one of the three does not have finite support, we have commutativity and associativity and can calculate freely to obtain $|h| *|g|=$ $|g| *|\rho| *|f|<+\infty$ for all $f \in \Gamma(\mathscr{G})$, proving that $h$ belongs to the second dual of $\{g\}$ for every $g \in \mathscr{G}$, hence to the second dual of $\mathscr{G}$.

Proposition 3.7 If $\mathscr{G}$ is a subset of $\mathscr{F}_{\text {finite }}(\mathbf{C}, \mathbf{C})$, the second dual $\Psi(\Psi(\mathscr{G}))=\Psi^{\circ 2}(\mathscr{G})$ always contains the set $\mathscr{G}^{[*]}$ :

$$
\Psi(\Psi(\mathscr{G}))=\Psi^{\circ 2}(\mathscr{G}) \supset \mathscr{G}^{[*]} .
$$

Proof If $h=g * \rho$ with $g \in \mathscr{G}$ and $\rho$ has finite support, then as in the previous proof we have commutativity and associativity and can calculate freely to obtain $h * g=(g * \rho) * f=(f * g) * \rho=0 * \rho=0$ for all $f \in \Psi(\mathscr{G})$, proving that $h$ belongs to the second dual of $\mathscr{G}$ under $\Psi$.

As we shall see in Examples 3.8-3.11, sometimes we have equality in (3.5) or (3.6), sometimes not. An example of strict inclusion is Theorem 4.1.

Example 3.8 Let us define $\mathscr{G}_{0}=\mathscr{F}_{\text {finite }}(G, \mathbf{C})$ and $\mathscr{G}_{1}=\mathscr{F}(G, \mathbf{C})$. Then $\Gamma\left(\mathscr{G}_{0}\right)=\mathscr{G}_{1}$ and $\Gamma\left(\mathscr{G}_{1}\right)=\mathscr{G}_{0}$; it follows that $\left(\Gamma^{\circ 2}\right)\left(\mathscr{G}_{j}\right)=\mathscr{G}_{j}, j=0$, 1 . So both $\mathscr{G}_{0}$ and $\mathscr{G}_{1}$ are closed for $\Gamma^{\circ 2}$.

We have $\Psi\left(\mathscr{G}_{0}\right)=\Psi\left(\mathscr{G}_{1}\right)=\{0\}$ and

$$
(\Psi \circ \Psi)\left(\mathscr{G}_{0}\right)=(\Psi \circ \Psi)\left(\mathscr{G}_{1}\right)=\mathscr{G}_{1}=\Gamma^{\circ 2}\left(\mathscr{G}_{1}\right) \supset \Gamma^{\circ 2}\left(\mathscr{G}_{0}\right)=\mathscr{G}_{0}
$$

with strict inclusion if and only if $G$ is infinite. 
Example 3.9 Take now $G=\mathbf{Z}^{n}$ and $\mathscr{G}=\left\{\delta_{0}\right\}$, a singleton set. Then $\Gamma(\mathscr{G})=$ $\mathscr{F}\left(\mathbf{Z}^{n}, \mathbf{C}\right)$, the space of all functions. It follows that $\Gamma^{\circ 2}(\mathscr{G})=\mathscr{F}_{\text {finite }}\left(\mathbf{Z}^{n}, \mathbf{C}\right)$. The higher powers are $\Gamma^{\circ k}(\mathscr{G})=\mathscr{F}\left(\mathbf{Z}^{n}, \mathbf{C}\right)$ for odd $k \geqslant 1$ and $\Gamma^{\circ k}(\mathscr{G})=\mathscr{F}_{\text {finite }}\left(\mathbf{Z}^{n}, \mathbf{C}\right)$ for even $k \geqslant 2$.

We see that $\Psi(\mathscr{G})=\{0\}$; the second dual is $\Psi^{\circ 2}(\mathscr{G})=\mathscr{F}\left(\mathbf{Z}^{n}, \mathbf{C}\right) \supsetneqq \mathscr{G}^{[*]}=$ $\mathscr{F}_{\text {finite }}\left(\mathbf{Z}^{n}, \mathbf{C}\right)=\Gamma^{\circ 2}(\mathscr{G})$.

Here $\Psi^{\circ 2}(\mathscr{G}) \supsetneqq \Gamma^{\circ 2}(\mathscr{G})$.

Example 3.10 Let $\mathscr{G}$ be the subfamily of $\mathscr{F}(G, \mathbf{C})$ whose only member is the constant function with value 1 . Then $\Gamma(\mathscr{G})=l^{1}(G)$. Moreover $\Gamma^{\circ 2}(\mathscr{G})=l^{\infty}(G)$, the family of all bounded functions. The higher powers are alternatively equal to $l^{1}(G)$ (odd $k \geqslant 1$ ) and $l^{\infty}(G)$ (even $k \geqslant 2$ ). So both $l^{1}(G)$ and $l^{\infty}(G)$ are fixed points for $\Gamma \circ$ $\Gamma$.

We have

$$
\Psi(\mathscr{G})=\left\{f \in l^{1}(G) ; \sum f(x)=0\right\}
$$

and

$$
\Psi^{\circ 2}(\mathscr{G})=\{h \in \mathscr{F}(G, \mathbf{C}) ; h \text { is constant }\}=\mathscr{G}^{[*]} \subset \Gamma^{\circ 2}(\mathscr{G})=l^{\infty}(G)
$$

with strict inclusion if and only if $G$ has more than one element. So here $\Psi^{\circ 2}(\mathscr{G}) \varsubsetneqq$ $\Gamma^{\circ 2}(\mathscr{G})$ unless $G$ has one element.

Example 3.11 Next, take $G=\mathbf{Z}$ and $\mathscr{G}=\left\{\delta_{-1}-\delta_{0}\right\}$ defining a difference operator. Then $\Gamma(\mathscr{G})=\mathscr{F}(\mathbf{Z}, \mathbf{C})$ and

$$
\Gamma^{\circ 2}(\mathscr{G})=\mathscr{F}_{\text {finite }}(\mathbf{Z}, \mathbf{C}) \supsetneqq \mathscr{G}^{[*]}=\left\{f \in \mathscr{F}_{\text {finite }}(\mathbf{Z}, \mathbf{C}) ; \sum f(x)<+\infty\right\} .
$$

We have

$$
\Psi(\mathscr{G})=\{f \in \mathscr{F}(\mathbf{Z}, \mathbf{C}) ; f \text { is constant }\}
$$

and

$$
\Psi^{\circ 2}(\mathscr{G})=\left\{h \in l^{1}(\mathbf{Z}) ; \sum h(x)=0\right\} \supsetneqq \mathscr{G}^{[*]} .
$$

Also here $\Psi^{\circ 2}(\mathscr{G}) \supsetneqq \Gamma^{\circ 2}(\mathscr{G})$.

\section{Affine Functions of a Real Variable}

A function $F: \mathbf{R} \rightarrow \mathbf{C}$ is said to be affine if it is of the form $F(x)=a x+b$ for some constants $a$ and $b$. Its restriction $f=\left.F\right|_{\mathbf{Z}}=F \circ j$ to $\mathbf{Z}$, where $j: \mathbf{Z} \rightarrow \mathbf{R}$ is the inclusion mapping, satisfies $f * \mu_{\mathrm{aff}}=0$, where $\mu_{\mathrm{aff}}=\delta_{-1}-2 \delta_{0}+\delta_{1}$. Conversely, if $f$ is defined on $\mathbf{Z}$, we can define an extension $F(x+t)=(1-t) f(x)+t f(x+1)$, 
$(x, t) \in \mathbf{Z} \times[0,1]$, which is affine between any two neighboring integers. If $f * \mu_{\text {aff }}=$ 0 , then $F$ is affine on the whole real line.

Let us define

$$
\begin{aligned}
& \mathscr{F}=\mathscr{F}(\mathbf{Z}, \mathbf{C}) ; \quad \mathscr{F}_{\text {finite }}=\mathscr{F}_{\text {finite }}(\mathbf{Z}, \mathbf{C}) \\
& \mathscr{M}=\left\{\mu_{\text {aff }}\right\}=\left\{\delta_{-1}-2 \delta_{0}+\delta_{1}\right\} ; \quad \mathscr{M}^{[*]}=\left\{g * \rho ; g \in \mathscr{M}, \rho \in \mathscr{F}_{\text {finite }}\right\} ; \\
& \mathscr{H}=\{h \in \mathscr{F} ; \sup |h(x)| /(1+|x|)<+\infty\} ; \\
& \mathscr{N}=\left\{v \in \mathscr{F} ; \sum|x v(x)|<+\infty\right\} ; \quad \mathscr{N}_{0}=\left\{v \in \mathscr{N} ; \sum v(x)=\sum x v(x)=0\right\} .
\end{aligned}
$$

Denote by $\mathscr{A}$ the family of all restrictions to $\mathbf{Z}$ of affine functions. Thus $\mathscr{A}$ is by definition equal to $\Psi(\mathscr{M})$.

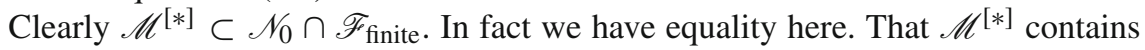
$\mathscr{N}_{0} \cap \mathscr{F}_{\text {finite }}$ can be proved using the Fourier transformation and classical results from complex analysis, but since we do not need this, I will not prove it here.

Concerning $\mathscr{N}$ and $\mathscr{N}_{0}$, we note that

$$
\mathscr{N}_{0} \subset \mathscr{N}=\mathscr{N}_{0}+\mathscr{F}_{\text {finite }} .
$$

In fact, if $f \in \mathscr{N}$, we can define $f_{0}=f+(a-b) \delta_{0}+b \delta_{1}$, so that $\sum f_{0}(x)=$ $\sum f(x)+a$, which is zero if $a=-\sum f(x)$, and $\sum x f_{0}(x)=\sum x f(x)+b$, which is zero if $b=-\sum x f(x)$. With these choices of $a$ and $b, f_{0}$ belongs to $\mathscr{N}_{0}$ and $f$ belongs to $\mathscr{N}_{0}+\mathscr{F}_{\text {finite }}$.

Theorem 4.1 The duals of $\mathscr{M}, \mathscr{A}, \mathscr{H}, \mathscr{N}$ and $\mathscr{N}_{0}$ under $\Gamma$ and $\Psi$ are:

1. $\Gamma(\mathscr{M})=\mathscr{F}$;

2. $\Psi(\mathscr{M})=\mathscr{A}$;

3. $\Gamma(\mathscr{A})=\mathscr{N}$;

4. $\Psi(\mathscr{A})=\mathscr{N}_{0}$;

5. $\Gamma(\mathscr{H})=\mathscr{N}$;

6. $\Psi(\mathscr{H})=\{0\}$;

7. $\Gamma(\mathscr{N})=\mathscr{H}$;

8. $\Psi(\mathscr{N})=\{0\}$;

9. $\Gamma\left(\mathscr{N}_{0}\right)=\mathscr{H}$;

10. $\Psi\left(\mathscr{N}_{0}\right)=\mathscr{A}$.

Proof Property 1 follows from the fact that $\mathscr{M} \subset \mathscr{F}_{\text {finite }}$, implying that $\Gamma(\mathscr{M}) \supset$ $\Gamma\left(\mathscr{F}_{\text {finite }}\right)=\mathscr{F}$.

Property 2 is the definition of $\mathscr{A}$.

Property 3: We have $\mathscr{A} \subset \mathscr{H}$ so that $\Gamma(\mathscr{A}) \supset \Gamma(\mathscr{H})=\mathscr{N}$ in view of 5 to be proved below. The inclusion $\mathscr{N} \subset \Gamma(\mathscr{A})$ is easy to see directly. Property 4 follows.

Properties 5 and 7 follow from Example 3.10: $\Gamma\left(l^{\infty}(\mathbf{Z})\right)=l^{1}(\mathbf{Z})$ and $\Gamma\left(l^{1}(\mathbf{Z})\right)=$ $l^{\infty}(\mathbf{Z})$. In fact, $h$ belongs to $\mathscr{H}$ if and only if $x \mapsto h(x) /(1+|x|)$ belongs to $l^{\infty}$ and $v$ belongs to $\mathscr{N}$ if and only if $x \mapsto(1+|x|) v(x)$ is in $l^{1}$, so that 5 and 7 are just the result from Example 3.10 with different weight functions. 
Property 6 is obvious.

For property 7 , see above on property 5 .

Property 8 is obvious.

On property 9: We have a priori $\Gamma\left(\mathscr{N}_{0}\right) \supset \Gamma(\mathscr{N})$, but in fact we have equality here since (4.1) implies that

$$
\Gamma\left(\mathscr{N}_{0}\right) \supset \Gamma(\mathscr{N})=\Gamma\left(\mathscr{N}_{0}+\mathscr{F}_{\text {finite }}\right)=\Gamma\left(\mathscr{N}_{0}\right) .
$$

Here the last equality is a consequence of the fact that functions with finite support never influence $\Gamma$.

10: Clearly $f * v=0$ if $f \in \mathscr{A}$ and $v$ belongs to $\mathscr{N}_{0}$, so $\mathscr{A} \subset \Gamma\left(\mathscr{N}_{0}\right)$. Since $\mathscr{N}_{0} \supset \mathscr{M}$, we have $\Psi\left(\mathscr{N}_{0}\right) \subset \Psi(\mathscr{M})=\mathscr{A}$ by property 2.

Corollary 4.2 The second duals of $\mathscr{M}, \mathscr{A}, \mathscr{H}, \mathscr{N}$ and $\mathscr{N}_{0}$ under $\Gamma$ and $\Psi$ are:

1. $(\Gamma \circ \Psi)(\mathscr{M})=\Gamma(\mathscr{A})=\mathscr{N}$;

2. $(\Gamma \circ \Gamma)(\mathscr{M})=\Gamma(\mathscr{F})=\mathscr{F}_{\text {finite; }}$;

3. $(\Psi \circ \Psi)(\mathscr{M})=\Psi(\mathscr{A})=\mathscr{N}_{0}$;

4. $(\Psi \circ \Gamma)(\mathscr{M})=\Psi(\mathscr{F})=\{0\}$;

5. $(\Gamma \circ \Psi)(\mathscr{A})=\Gamma\left(\mathscr{N}_{0}\right)=\mathscr{H}$;

6. $(\Gamma \circ \Gamma)(\mathscr{A})=\Gamma(\mathscr{N})=\mathscr{H}$;

7. $(\Psi \circ \Psi)(\mathscr{A})=\Psi\left(\mathscr{N}_{0}\right)=\mathscr{A}$;

8. $(\Psi \circ \Gamma)(\mathscr{A})=\Psi(\mathscr{N})=\{0\}$;

9. $(\Gamma \circ \Psi)(\mathscr{H})=\Gamma(\{0\})=\mathscr{F}$;

10. $(\Gamma \circ \Gamma)(\mathscr{H})=\Gamma(\mathscr{N})=\mathscr{H}$;

11. $(\Psi \circ \Psi)(\mathscr{H})=\Psi(\{0\})=\mathscr{F}$;

12. $(\Psi \circ \Gamma)(\mathscr{H})=\Psi(\mathscr{N})=\{0\}$;

13. $(\Gamma \circ \Psi)(\mathscr{N})=\Gamma(\{0\})=\mathscr{F}$;

14. $(\Gamma \circ \Gamma)(\mathscr{N})=\Gamma(\mathscr{H})=\mathscr{N}$;

15. $(\Psi \circ \Psi)(\mathscr{N})=\Psi(\{0\})=\mathscr{F}$;

16. $(\Psi \circ \Gamma)(\mathscr{N})=\Psi(\mathscr{H})=\{0\}$;

17. $(\Gamma \circ \Psi)\left(\mathscr{N}_{0}\right)=\Gamma(\mathscr{A})=\mathscr{N}$;

18. $(\Gamma \circ \Gamma)\left(\mathscr{N}_{0}\right)=\Gamma(\mathscr{H})=\mathscr{N}$;

19. $(\Psi \circ \Psi)\left(\mathscr{N}_{0}\right)=\Psi(\mathscr{A})=\mathscr{N}_{0}$;

20. $(\Psi \circ \Gamma)\left(\mathscr{N}_{0}\right)=\Psi(\mathscr{H})=\{0\}$.

Proof These twenty properties are easy consequences of Theorem 4.1.

The corollary shows that, for $\Gamma \circ \Gamma, \mathscr{H}$ and $\mathscr{N}$ are closed, while $\mathscr{M}, \mathscr{A}$ and $\mathscr{N}_{0}$ are not. For $\Psi \circ \Psi, \mathscr{A}$ and $\mathscr{N}_{0}$ are closed, while $\mathscr{M}, \mathscr{H}$ and $\mathscr{N}$ are not closed.

\section{Affine Functions of a Complex Variable}

A function $F: \mathbf{Z}[\mathrm{i}] \rightarrow \mathbf{C}$ is said to be affine if it is of the form $F(z)=a z+b \bar{z}+c$ for some constants $a, b, c$. We now redefine $\mathscr{M}$ and the other classes in the previous 
section:

$$
\begin{aligned}
& \mathscr{F}=\mathscr{F}(\mathbf{Z}[\mathrm{i}], \mathbf{C}) ; \quad \mathscr{F}_{\text {finite }}=\mathscr{F}_{\text {finite }}(\mathbf{Z}[\mathrm{i}], \mathbf{C}) \\
& \mathscr{M}=\left\{\mu_{1}, \mu_{2}, \mu_{3}\right\} \text {, where } \\
& \mu_{1}=\delta_{-1-i}-\delta_{-1}-\delta_{-i}+\delta_{0}, \mu_{2}=\delta_{-1}-2 \delta_{0}+\delta_{1}, \\
& \text { and } \mu_{3}=\delta_{-\mathrm{i}}-2 \delta_{0}+\delta_{\mathrm{i}} \text {; } \\
& \mathscr{M}^{[*]}=\left\{g * \rho ; g \in \mathscr{M}, \rho \in \mathscr{F}_{\text {finite }}\right\} \text {; } \\
& \mathscr{H}=\{h \in \mathscr{F} ; \sup |h(z)| /(1+|z|)<+\infty\} ; \\
& \mathscr{N}=\left\{v ; \sum|z \rho(z)|<+\infty\right\} ; \\
& \mathscr{N}_{0}=\left\{v \in \mathscr{N} ; \sum v(z)=\sum z v(z)=\sum \bar{z} v(z)=0\right\} \text {. }
\end{aligned}
$$

Let us also redefine $\mathscr{A}$ to be the set of restrictions to $\mathbf{Z}[\mathrm{i}]$ of affine functions on $\mathbf{C}$. Thus $\mathscr{A}=\Psi(\mathscr{M})$ by definition.

We note that, as before $\mathscr{N}=\mathscr{N}_{0}+\mathscr{F}_{\text {finite }}$.

It is easy to see that if a function $F: \mathbf{C} \rightarrow \mathbf{C}$ is affine, then its restriction $f$ to $\mathbf{Z}$ [i] satisfies $f * \mu_{j}=0, j=1,2,3$.

If $f: \mathbf{Z}[\mathrm{i}] \rightarrow \mathbf{C}$, we define an extension to $\mathbf{C}$ by putting

$$
\begin{aligned}
F(s x+t y)= & (1-s) f(x+\mathrm{i} y)+s f(x+\mathrm{i} y+1) \\
& +(1-t) f(x+\mathrm{i} y)+t f(x+\mathrm{i} y+\mathrm{i})),
\end{aligned}
$$

where $(x+\mathrm{i} y, s, t) \in \mathbf{Z}[\mathrm{i}] \times[0,1] \times[0,1]$. If $f * \mu_{1}=0$, then this extension is affine in each square with vertices in $z, z+1, z+1+\mathrm{i}, z+\mathrm{i}$. If $f$ also satisfies $f * \mu_{2}=f * \mu_{3}=0$, then the extension is affine in all of $\mathbf{C}$.

Theorem 5.1 The duals of $\mathscr{M}, \mathscr{A}, \mathscr{N}, \mathscr{N}_{0}$ and $\mathscr{H}$ under $\Gamma$ and $\Psi$ are, with the new definitions introduced here, just as in Theorem 4.1.

Proof We can argue just as in the proof of Theorem 4.1.

Corollary 5.2 The second duals of $\mathscr{M}, \mathscr{A}, \mathscr{N}, \mathscr{N}_{0}$ and $\mathscr{H}$ under $\Gamma$ and $\Psi$ are just as in Corollary 4.2.

Concerning closed and not closed families under $\Gamma \circ \Gamma$ and $\Psi \circ \Psi$, the situation is the same as mentioned after the proof of Corollary 4.2.

\section{Approaching Monodiffric Functions via Polygons}

In analogy with the result that an integral $\int_{C} h(z) \mathrm{d} z$ over a closed curve vanishes if $h$ is holomorphic inside $C$, we shall look at integrals over a simple closed polygon $P$ in the complex plane $\mathbf{C}$.

Take $P$ consisting of $m$ segments $\left[a_{0}, a_{1}\right],\left[a_{1}, a_{2}\right], \ldots,\left[a_{m-1}, a_{0}\right]$, where $a_{0}, \ldots, a_{m-1}$ are given points in $\mathbf{C}$. The polygon is thus determined by $\left(a_{0}, \ldots, a_{m-1}\right) \in$ 
$\mathbf{C}^{m}$. We shall say that a complex-valued function $f$ defined on $P$ is piecewise affine if $f$ is affine on each segment $\left[a_{j}, a_{j+1}\right]$. This means that, if $a_{j+1} \neq a_{j}$,

$$
f(z)=\frac{a_{j+1}-z}{a_{j+1}-a_{j}} f\left(a_{j}\right)+\frac{z-a_{j}}{a_{j+1}-a_{j}} f\left(a_{j+1}\right), \quad z \in\left[a_{j}, a_{j+1}\right], \quad j=0, \ldots, m-1 .
$$

Here and in the sequel we count indices modulo $m$. If $a_{j+1}=a_{j}$ the formula reduces to $f(z)=f\left(a_{j}\right)=f\left(a_{j+1}\right)$.

The integral of a piecewise affine function is easy to calculate.

Proposition 6.1 Let $f$ be piecewise affine on a closed polygon $P$ determined by $\left(a_{0}, \ldots, a_{m-1}\right)$. Then

$$
\int_{P} f(z) \mathrm{d} z=\frac{1}{2} \sum_{0}^{m-1} f\left(a_{j}\right)\left(a_{j+1}-a_{j-1}\right)=\frac{1}{2} \sum_{0}^{m-1}\left(f\left(a_{j-1}\right)-f\left(a_{j+1}\right)\right) a_{j} .
$$

Proof If $f$ is affine on a segment $\left[a_{j}, a_{j+1}\right]$ we can replace it by its average over the segment without changing the value of the integral. The average is $\frac{1}{2} f\left(a_{j}\right)+\frac{1}{2} f\left(a_{j+1}\right)$. This implies that

$$
\begin{aligned}
\int_{a_{j}}^{a_{j+1}} f(z) \mathrm{d} z & =\int_{a_{j}}^{a_{j+1}}\left(\frac{1}{2} f\left(a_{j}\right)+\frac{1}{2} f\left(a_{j+1}\right)\right) \mathrm{d} z \\
& =\left(\frac{1}{2} f\left(a_{j}\right)+\frac{1}{2} f\left(a_{j+1}\right)\right)\left(a_{j+1}-a_{j}\right)
\end{aligned}
$$

To finish we just need to sum over $j$ and change the indices.

Definition 6.2 Given a simple closed polygon $P$ in $\mathbf{C}$ we shall say that a function $f$ defined on its vertices is holomorphic inside $P$ if $\int_{P} f_{\text {aff }}(z) \mathrm{d} z=0$, where $f_{\text {aff }}$ is the unique piecewise affine function on $P$ which takes the same values as $f$ on the vertices.

Proposition 6.1 now yields the following result.

Corollary 6.3 Let $a_{0}, \ldots, a_{m-1}$ be $m$ points in the complex plane. A function defined on the vertices of the closed polygon $P$ defined by $\left(a_{0}, \ldots, a_{m-1}\right)$ is holomorphic inside $P$ if and only if

$$
\sum_{0}^{m-1} f\left(a_{j}\right)\left(a_{j+1}-a_{j-1}\right)=0
$$

When $m=1,2$, every function is holomorphic.

When $m=3$ and $\left(a_{0}, a_{1}, a_{2}\right)=(a, b, c)$, the condition becomes

$$
f(a)(b-c)+f(b)(c-a)+f(c)(a-b)=0,
$$


which can be written as

$$
\frac{f(b)-f(a)}{b-a}=\frac{f(c)-f(a)}{c-a} .
$$

This means that the difference quotient is the same in the direction from $a$ to $b$ as in the direction from $a$ to $c$. In particular, if $b=a+1$ and $c=a+\mathrm{i}$, we get

$$
\frac{f(a+1)-f(a)}{1}=\frac{f(a+\mathrm{i})-f(a)}{\mathrm{i}} .
$$

Definition 6.4 Let $A$ be a subset of $\mathbf{Z}$ [i] which is a union of triples $z, z+1, z+\mathrm{i}$, $z \in \mathbf{Z}[\mathrm{i}]$. A complex-valued function $f$ defined on $A$ shall be said to be monodiffric of the first kind or holomorphic in the sense of Isaacs if (6.2) holds for all $a \in A$ such that also $a+1$ and $a+\mathrm{i}$ belong to $A$.

Isaacs $[6,7]$ studied functions that satisfy the equality (6.2). They satisfy $\mu_{\mathrm{I}} * f=0$, where

$$
\mu_{\mathrm{I}}=\delta_{-1}+\mathrm{i} \delta_{-\mathrm{i}}-(1+\mathrm{i}) \delta_{0}
$$

We shall denote by $\mathscr{O}_{\mathrm{I}}(A)$ the set of functions $f: A \rightarrow \mathbf{C}$ which satisfy $\mu_{\mathrm{I}} * f=0$ at the points where the convolution product is defined, the functions holomorphic in the sense of Isaacs.

When $m=4$ and $\left(a_{0}, a_{1}, a_{2}, a_{3}\right)=(a, b, c, d)$, condition (6.1) becomes

$$
f(a)(b-d)+f(b)(c-a)+f(c)(d-b)+f(d)(a-c)=0,
$$

which may be written

$$
\frac{f(c)-f(a)}{c-a}=\frac{f(d)-f(b)}{d-b}
$$

meaning that the difference quotient in the direction from $a$ to $c$ is equal to that in the direction from $b$ to $d$. This is the definition studied by Ferrand [5]. In particular, if we let $b=a+1, c=a+1+\mathrm{i}$ and $d=a+\mathrm{i}$, we get

$$
\frac{f(a+1+\mathrm{i})-f(a)}{1+\mathrm{i}}=\frac{f(a+\mathrm{i})-f(a+1)}{\mathrm{i}-1} .
$$

Definition 6.5 Let $A$ be a subset of $\mathbf{Z}[\mathrm{i}]$ which is a union of quadruples $z, z+1$, $z+1+\mathrm{i}, z+\mathrm{i}$. A function $f: A \rightarrow \mathbf{C}$ is said to be monodiffric of the second kind or holomorphic in the sense of Ferrand if (6.4) holds for all $a \in A$ such that also $a+1$, $a+\mathrm{i}$, and $a+1+\mathrm{i}$ all belong to $A$.

Functions satisfying (6.4) satisfy $\mu_{\mathrm{F}} * f=0$, where

$$
\mu_{\mathrm{F}}=\delta_{-1-\mathrm{i}}+\mathrm{i} \delta_{-\mathrm{i}}-\mathrm{i} \delta_{-1}-\delta_{0}
$$


We shall denote by $\mathscr{O}_{\mathrm{F}}(A)$ the set of functions $f: A \rightarrow \mathbf{C}$ which satisfy $\mu_{\mathrm{F}} * f=0$ where the convolution product is defined, the functions holomorphic in the sense of Ferrand.

\section{Cauchy Problems for Functions Holomorphic in the Sense of Isaacs}

We can solve uniquely a Cauchy problem with data given on an infinite set:

Theorem 7.1 Given any functions $\varphi: \mathbf{Z} \rightarrow \mathbf{C}$ and $\psi:-\mathbf{N} \rightarrow \mathbf{C}$ with $\varphi(0)=\psi(0)$, there exists a function $f \in \mathscr{O}_{\mathrm{I}}(\mathbf{Z}[\mathrm{i}])$ such that $f(x)=\varphi(x), x \in \mathbf{Z}$ and $f(\mathrm{i} y)=\psi(y)$ for $y \in-\mathbf{N}$.

Proof We define $f(x)=\varphi(x)$ for $x \in \mathbf{Z}$ and then $f(x+\mathrm{i})$ as required by the condition given by the difference operator $\mu_{\mathrm{I}}$. This means that we must have $f(x+\mathrm{i})-f(x)=$ $-\mathrm{i}(f(x+1)-f(x))$, implying that we must take $f(x+\mathrm{i})=-\mathrm{i} \varphi(x+1)+(1+\mathrm{i}) \varphi(x)$. Continuing in this way, we see that $f(z)$ is uniquely determined for $z=x+\mathrm{i} y, y \geqslant 0$.

For $f(x+\mathrm{i} y)$ with $y \leqslant-1$ we proceed in the same way from the values of $\psi$, now going to the right and left from $x=0, y \leqslant 0$, and then downwards.

In particular this theorem shows that there are holomorphic functions in the sense of Isaacs which grow arbitrarily fast on the real axis, and in fact also in other sectors.

Proposition 7.2 If $\varphi, \psi>0$, then the solution $f \in \mathscr{O}_{\mathrm{I}}(\mathbf{Z}[\mathrm{i}])$ to the Cauchy problem in Theorem 7.1 satisfies in the upper half plane

$$
|f(x+\mathrm{i} y)| \geqslant \varphi(x)+\varphi(x+y), \quad(x, y) \in \mathbf{Z} \times \mathbf{N}
$$

in the third quadrant

$$
2^{|y| / 2}|f(x+\mathrm{i} y)| \geqslant \varphi(x)+\psi(y), \quad(x, y) \in(-\mathbf{N}) \times(-\mathbf{N}) ;
$$

in the fourth quadrant

$$
\begin{gathered}
|f(x+\mathrm{i} y)| \geqslant \psi(y)+\psi(x+y), \quad(x, y) \in \mathbf{N} \times(-\mathbf{N}), x+y \leqslant 0 ; \text { and } \\
|f(x+\mathrm{i} y)| \geqslant \psi(y)+\varphi(x+y), \quad(x, y) \in \mathbf{N} \times(-\mathbf{N}), x+y \geqslant 0 .
\end{gathered}
$$

Proof The induction follows different directions in the various sectors, which is why we shall estimate different terms in (6.2) by the other terms. Let us introduce a new function $g(x+\mathrm{i} y)=\mathrm{i}^{-a x-b y} f(x+\mathrm{i} y)$ and see what (6.2) means for $g$. The result is

$$
\mathrm{i}^{a} g(z+1)+\sqrt{2} \mathrm{i}^{-3 / 2} g(z)+\mathrm{i}^{b+1} g(z+\mathrm{i})=0 .
$$

In the upper half plane we choose $a=-3 / 2$ and $b=-1 / 2$ and get $g(z+\mathrm{i})=$ $g(z+1)+\sqrt{2} g(z)$, an estimate of $g$ at points with $\operatorname{Im} z>\operatorname{Im}(z+1)=\operatorname{Im} z$. This implies (7.1) since $|f|=|g|$. 
In the third quadrant we choose $a=1 / 2$ and $b=-1 / 2$ and get

$$
\sqrt{2} g(z)=g(z+1)+g(z+\mathrm{i})
$$

giving us an estimate of $g(z)$ by the value at two points $z+1$ and $z+\mathrm{i}$ with $\operatorname{Re} z+\operatorname{Im} z<$ $\operatorname{Re}(z+1)+\operatorname{Im}(z+1)=\operatorname{Re}(z+\mathrm{i})+\operatorname{Im}(z+\mathrm{i})$. (The induction goes along decreasing values of $\operatorname{Re} z+\operatorname{Im} z$.) This implies (7.2) since $|f|=|g|$.

In the fourth quadrant, finally, we take $a=1 / 2$ and $b=3 / 2$, and obtain $g(z+\mathrm{i})=$ $g(z+1)+\sqrt{2} g(z)$, resulting in an estimate of $g(z+\mathrm{i})$ by the value at two points $z+1$ and $z$ with $\operatorname{Im}(z+1)-\operatorname{Re}(z+1)<\operatorname{Im} z-\operatorname{Re} z=\operatorname{Im}(z+\mathrm{i})-\operatorname{Re}(z+\mathrm{i})$. (Here the induction goes along decreasing values of $\operatorname{Im} z-\operatorname{Re} z$.) Hence (7.3) and (7.4).

It is crucial here that $|f(x+\mathrm{i} y)|$ tends to plus infinity as $|x| \vee|y| \rightarrow+\infty$ and not only as $|x| \wedge|y| \rightarrow+\infty$. In fact, the construction in later proofs will fail if $|f(x+\mathrm{i} y)|$ does not tend to $+\infty$ in a sequence of points $x+\mathrm{i} y$ such that $y$ remains bounded. We shall therefore estimate $|f(x+\mathrm{i} y)|$ from below by a function of $|x| \vee|y|$ which tends to plus infinity as $|x| \vee|y| \rightarrow+\infty$, equivalently as $|z| \rightarrow+\infty$.

Definition 7.3 Define two functions $\varphi_{0}$ and $\psi_{0}$ of $\varphi$ and $\psi$ by

$$
\varphi_{0}(x)=\inf _{|s| \geqslant|x|} \varphi(s), \quad x \in \mathbf{Z} ; \quad \psi_{0}(y)=\inf _{t \leqslant-|y|} \psi(t), \quad y \in \mathbf{Z}
$$

They are symmetric minorants of $\varphi$ and $\psi$ and tend to $+\infty$ as $|x| \vee|y|$ tends to $+\infty$ provided $\varphi$ and $\psi$ do so.

We shall estimate $|f|$ from below by $\tau(z)=\tau(x+\mathrm{i} y)=\varphi_{0}(|x| \vee|y|) \wedge \psi_{0}(|x| \vee|y|)$, which also tends to $+\infty$ as $|z| \rightarrow+\infty$ if both $\varphi$ and $\psi$ do so. The arguments for the Cauchy data are $x, x+y$ and $y$. We have to relate them to $|x| \vee|y|$, to be done differently in various sectors.

Proposition 7.4 We consider eight sectors in the plane:

1. In the first quadrant, $|x| \vee|y|$ is equal to $x \vee y \leqslant x+y$, so that $\varphi(x+y) \geqslant \varphi_{0}(|x| \vee$ $|y|) \geqslant \tau(z)$.

2.1. In the sector defined by $0 \leqslant-2 x \leqslant y,|x| \vee|y|$ is equal to $y$ and $x+y \geqslant \frac{1}{2} y$, so that $\varphi(x+y) \geqslant \varphi_{0}\left(\frac{1}{2} y\right)=\varphi_{0}\left(\frac{1}{2}(|x| \vee|y|)\right) \geqslant \tau\left(\frac{1}{2} z\right)$;

2.2. In the sector defined by $0 \leqslant y \leqslant-2 x,|x| \vee|y| \leqslant|x| \vee 2|x|=2|x|$ since now $|x| \geqslant \frac{1}{2} y$, implying that $\varphi(x) \geqslant \varphi_{0}(|x|) \geqslant \varphi_{0}\left(\frac{1}{2}(|x| \vee|y|)\right) \geqslant \tau\left(\frac{1}{2} z\right) ;$

3.1. In the sector defined by $x \leqslant y \leqslant 0,|x| \vee|y|$ is equal to $-x=|x|$, so that we have $\varphi(x) \geqslant \varphi_{0}(|x|)=\varphi_{0}(|x| \vee|y|) \geqslant \tau(z)$;

3.2. In the sector defined by $y \leqslant x \leqslant 0,|x| \vee|y|$ is equal to $-y=|y|$, so that $\psi(y) \geqslant \psi_{0}(|y|)=\psi_{0}(|x| \vee|y|) \geqslant \tau(z) ;$

4.1. In the sector defined by $y \leqslant-x \leqslant 0,|x| \vee|y|$ is equal to $|y|$, so that $\psi(y)=\psi_{0}(|x| \vee|y|) \geqslant \tau(z)$;

4.2. In the sector defined by $-x \leqslant y \leqslant-\frac{1}{2} x,|x| \vee|y|$ is equal to $x$, so that $\psi(y)=\psi_{0}(|y|) \geqslant \psi_{0}\left(\frac{1}{2} x\right)=\psi_{0}\left(\frac{1}{2}(|x| \vee|y|)\right) \geqslant \tau\left(\frac{1}{2} z\right) ;$ 
4.3. In the sector defined by $-\frac{1}{2} x \leqslant y \leqslant 0$, finally, $|x| \vee|y|$ is equal to $x$ and $x+$ $y \geqslant \frac{1}{2} x=\frac{1}{2}(|x| \vee|y|)$, implying that $\varphi(x+y) \geqslant \varphi_{0}(|x+y|) \geqslant \varphi\left(\frac{1}{2}(|x| \vee\right.$ $|y|)) \geqslant \tau\left(\frac{1}{2} z\right)$.

Proof In each of the eight cases, simple comparisons give the result.

Theorem 7.5 Functions in $\mathscr{O}_{\mathrm{I}}(\mathbf{C})$ can grow as fast as we want.

Proof Because of the weaker estimate (7.2) in the third quadrant we replace $\varphi_{0}$ in sector 3.1 in Proposition 7.4 by $\varphi_{1}(x)=2^{|x| / 2} \varphi_{0}(x)$. In sector 3.2 we replace instead $\psi_{0}$ by $\psi_{1}(y)=2^{|y| / 2} \psi_{0}(y)$. This proves that $\tau(z)$ tends to $+\infty$ as $|z| \rightarrow+\infty \ldots$ as fast as we like.

Theorem 7.6 The second dual under $\Psi$ of $\left\{\mu_{\mathrm{I}}\right\}$ is

$$
\Psi^{\circ 2}\left(\left\{\mu_{\mathrm{I}}\right\}\right)=\left\{\mu_{\mathrm{I}} * \rho ; \rho \in \mathscr{F}_{\text {finite }}(\mathbf{C}, \mathbf{C})\right\}=\mu_{\mathrm{I}}^{[*]} .
$$

Thus the class $\mu_{\mathrm{I}}{ }^{[*]}$ is closed for $\Psi^{\circ 2}$, in contrast to the situation in Theorems 4.1 and 5.1, where $\Psi^{\circ 2}(\mathscr{M})=\mathscr{N}_{0} \supsetneqq \mathscr{M}$ (see property 3 in Corollaries 4.2 and 5.2).

Proof From Proposition 3.7 it follows that the second dual contains $\mu_{\mathrm{I}}{ }^{[*]}$.

For the converse, suppose that there are infinitely many points $z$ with $\rho(z) \neq 0$. We then take $f \in \mathscr{O}_{\mathrm{I}}(\mathbf{Z}[\mathrm{i}])$ so that $|f(z)| \geqslant 1 /|\rho(z)|$ for infinitely many points $z$ with $\rho(z) \neq 0$, preventing $|f| *|\rho|$ from converging.

\section{Cauchy Problems for Functions Holomorphic in the Sense of Ferrand}

Also the family $\mathscr{O}_{\mathrm{F}}(\mathbf{Z}[\mathrm{i}])$ is rich in fast-growing functions:

Theorem 8.1 Given any functions $\varphi: \mathbf{Z} \rightarrow \mathbf{C}$ and $\psi: \mathbf{Z} \rightarrow \mathbf{C}$ with $\varphi(0)=\psi(0)$, there exists a function $f \in \mathscr{O}_{\mathrm{F}}(\mathbf{Z}[\mathrm{i}])$ such that $f(x)=\varphi(x), x \in \mathbf{Z}$ and $f(\mathrm{i} y)=\psi(y)$, $y \in \mathbf{Z}$.

Proof In the first quadrant we define $f(x)=\varphi(x)$ for $x \in \mathbf{N}$ and $f(i y)=\psi(y)$, $y \in \mathbf{N}$ and then $f(1+\mathrm{i})$ as required by the condition given by the difference operator $\mu_{\mathrm{F}}$. We then go on: if we have already found the values of $f$ at three points $x+\mathrm{i} y$, $x+\mathrm{i} y+1$ and $x+\mathrm{i} y+\mathrm{i}$, then there is a unique value for $f$ at $x+\mathrm{i} y+1+\mathrm{i}$ which satisfies the condition.

Similary we can go on defining $f$ in each of the other quadrants, always going from three vertices of a square to the fourth, the one which is farthest from the origin.

In particular Theorem 8.1 shows that there are holomorphic functions in the sense of Ferrand which grow arbitrarily fast on the axes, and in fact also in other sectors.

Theorem 8.2 If $\varphi, \psi>0$, the function constructed in Theorem 8.1 satisfies

$$
|f(x+\mathrm{i} y)| \geqslant \varphi(x)+\psi(y), \quad(x, y) \in \mathbf{Z} \times \mathbf{Z} .
$$


Proof We define $g(x+\mathrm{i} y)=\mathrm{i}^{x-y} f(x+\mathrm{i} y)$, so that $g$ satisfies

$$
g(z+1+\mathrm{i})=g(z)+g(z+i)+g(z+1)
$$

in the first quadrant. This proves that $|f(x+\mathrm{i} y)|=g(x+\mathrm{i} y) \geqslant \varphi(x)+\psi(y)$, $x, y \in \mathbf{N}$, thus arbitrarily large for convenient choices of the Cauchy data. Because of the symmetry, similar estmates hold for the three other quadrants.

Definition 8.3 We keep the notation $\varphi_{0}$ from Definition 7.3 and redefine $\psi_{0}$ as

$$
\psi_{0}(y)=\inf _{|t| \geqslant|y|} \psi(t), \quad y \in \mathbf{Z}
$$

Proposition 8.4 Here we consider four sectors only.

1. In the sectors defined by $|y| \leqslant|x|,|x| \vee|y|$ is equal to $|x|$, so that $\varphi(x) \geqslant \varphi_{0}(|x| \vee|y|) \geqslant \tau(z)$.

2. In the sectors defined by $|x| \leqslant|y|,|x| \vee|y|$ is equal to $|y|$, so that $\psi(y) \geqslant \psi_{0}(|x| \vee|y|) \geqslant \tau(z)$.

Proof Simple comparisons will do.

Theorem 8.5 Functions in $\mathscr{O}_{\mathrm{F}}(\mathbf{C})$ can grow as fast as we want.

Proof This is clear in view of Proposition 8.4.

Theorem 8.6 The second dual under $\Psi$ of $\left\{\mu_{\mathrm{F}}\right\}$ is

$$
\Psi^{\circ 2}\left(\left\{\mu_{\mathrm{F}}\right\}\right)=\left\{\mu_{\mathrm{F}} * \rho ; \rho \in \mathscr{F}_{\text {finite }}(\mathbf{C}, \mathbf{C})\right\}=\mu_{\mathrm{F}}{ }^{[*]} .
$$

Thus the class $\mu_{\mathrm{F}}^{[*]}$ is closed for $(\Psi)^{\circ 2}$ also in this case.

Proof As before it is clear that the second dual contains $\mu_{\mathrm{F}}{ }^{[*]}$.

For the converse, suppose that there are infinitely many points $z$ with $\rho(z) \neq 0$. We then take $f \in \mathscr{O}_{\mathrm{F}}(\mathbf{Z}[\mathrm{i}])$ so that $|f(z)| \geqslant 1 /|\rho(z)|$ for infinitely many points $z$ with $\rho(z) \neq 0$, preventing $|\rho| *|f|$ from converging.

Since it may happen that $\rho$ is different from zero only in points $x+\mathrm{i} y$ with $y$ bounded, it is important here to be able to choose $f$ large also at such points; cf. Propositions 7.4 and 8.4.

Acknowledgements It was a privilege and a pleasure to participate in the conference in 2004 in honor of Carlos Alberto Berenstein. I am most grateful for the invitation by Daniele Struppa and Irene Sabadini to contribute to the present publication, now dedicated, in sadness, to the memory of Carlos. Lawrence Gruman provided essential corrections to a former version of Proposition 7.4. Sincere thanks!

Funding Open access funding provided by Uppsala University. 
Open Access This article is licensed under a Creative Commons Attribution 4.0 International License, which permits use, sharing, adaptation, distribution and reproduction in any medium or format, as long as you give appropriate credit to the original author(s) and the source, provide a link to the Creative Commons licence, and indicate if changes were made. The images or other third party material in this article are included in the article's Creative Commons licence, unless indicated otherwise in a credit line to the material. If material is not included in the article's Creative Commons licence and your intended use is not permitted by statutory regulation or exceeds the permitted use, you will need to obtain permission directly from the copyright holder. To view a copy of this licence, visit http://creativecommons.org/licenses/by/4.0/.

\section{References}

1. Benjamini, I., Lovász, L.: Harmonic and analytic functions on graphs. J. Geom. 76, 2-15 (2003)

2. Bourbaki, N.: Éléments de mathématique. Théorie des ensembles. Chapitres I et II. Hermann \& Cie, Paris (1954)

3. Cohn, P.M.: Universal Algebra, xv + 412 pp, 2nd edn. D. Reidel Publishing Co, Dortrecht (1981)

4. Duffin, R.J.: Basic properties of discrete analytic functions. Duke Math. J. 23, 335-363 (1956)

5. Ferrand, J.: Fonctions préharmoniques et fonctions préholomorphes. Bull. Sci. Math. 68, 152-180 (1944)

6. Isaacs, R.P.: A finite difference function theory. Univ. Nac. Tucumán. Revista A. 2, 177-201 (1941)

7. Isaacs, R.P.: Monodiffric functions. In: Construction and Applications of Conformal Maps: Proceedings of a Symposium, pp. 257-266. National Bureau of Standards Appl. Math. Series, No. 18. Washington, DC: U.S. Government Printing Office (1952)

8. Kenyon, R.: Conformal invariance of domino tiling. Ann. Probab. 28, 759-795 (2000)

9. Kiselman, C.O.: Functions on discrete sets holomorphic in the sense of Isaacs, or monodiffric functions of the first kind. Sci. in China Ser. A Math. 48 (Supplement), 86-96 (2005)

10. Kiselman, C.O.: Functions on discrete sets holomorphic in the sense of Ferrand, or monodiffric functions of the second kind. Sci. China Ser. A Math. 51(4), 604-619 (2008)

11. Kiselman, C.O.: Generalized convexity: The case of lineally convex Hartogs domains. Ann. Polon. Math. 123(1), 319-344 (2019)

12. Kiselman, C.O.: MS. Duality of convolution operators: a tool for shape analysis. Manuscript, submitted 2019 April 30 (2019)

13. Kiselman, C.O.: MS. Digital Geometry, Mathematical Morphology, and Discrete Optimization. Manuscript, xii + 449 pp (2020)

14. Курош, Александр Геннадиевич. 1962. Лекции по общей алгебре, 396 pp. Moscow: Государственное издательство физико-математической литературы

15. Lovász, L.: Discrete analytic functions: an exposition. Manuscript (2000). https://www.google.com/ search?client=firefox-b-e\&q=lovasz+analytic.pdf. Retrieved 03 Aug 2020

16. Martineau, A.: Sur les fonctionnelles analytiques et la transformation de Fourier-Borel. J. Anal. Math. 11, 1-164 (1963)

17. Nakamura, A., Rosenfeld, A.: Digital calculus. Inform. Sci. 98, 93-98 (1997)

18. Euvres de André Martineau: Paris: Éditions du Centre national de la Recherche scientifique (1977)

19. Serra, J.: Image Analysis and Mathematical Morphology. Academic Press, London (1982)

20. Serra, J. (ed.): Image Analysis and Mathematical Morphology. Volume 2: Theoretical Advances. Academic Press, London (1988)

Publisher's Note Springer Nature remains neutral with regard to jurisdictional claims in published maps and institutional affiliations. 